\title{
An Alternative Description for the Electronegativity Difference in Binary Ionic Solids
}

\author{
Heveson Lima, ${ }^{\oplus *, a}$ Douglas Ferreira, ${ }^{a}$ Adelmo S. Souza $a^{b}$ and Marcos A. C. dos Santos ${ }^{\circledR c}$ \\ ${ }^{a}$ Centro Multidisciplinar do Campus de Luís Eduardo Magalhães, \\ Universidade Federal do Oeste da Bahia, 47850-00 Luís Eduardo Magalhães-BA, Brazil \\ ${ }^{b}$ Centro Multidisciplinar do Campus de Bom Jesus da Lapa, \\ Universidade Federal do Oeste da Bahia, 47600-00 Bom Jesus da Lapa-BA, Brazil \\ ${ }^{c}$ Departamento de Física, Universidade Federal de Sergipe, 49100-00 São Cristóvão-SE, Brazil
}

\begin{abstract}
We are introducing an alternative analytical expression to the electronegativity difference $(\Delta \chi)$ as a function of the charge ge, $\mathrm{g}$ is the charge factor, the fraction of the electronic charge devoted to the bond and e the elementary charge, the packing factor $(\mathrm{p})$ and the effective atomic number $\left(\mathrm{Z}_{\text {eff }}\right)$ of binary ionic solids, by using the very basic Coulomb interaction, modified by the introduction of $\mathrm{p}$, and the relationship between the electric dipole moment $(\mu)$ and $\Delta \chi$ in Debye units. When compared to the Pauling's, Gordy's, Allred-Rochow's and Allen's scales, our calculations deviate around $10 \%$ to all ionic crystals with such data available in the literature. A very simple expression with satisfactory estimates, with no need of numerical procedure, is announced. The values of $g$ play the important role of indicating the character of the chemical bond. It opens up an alternative opportunity to understand the nature of ionic chemical bonds and is able to describe the character of the bonding in any ionic polyatomic system.
\end{abstract}

Keywords: binary ionic solids, Coulomb interaction, effective atomic number, charge transferred, chemical bond, electronegativity difference

\section{Introduction}

The nature of the chemical bond has been studied since the beginning of the $20^{\text {th }}$ century. Pauling ${ }^{1}$ and Mulliken ${ }^{2}$ gave the greatest contributions to the fundamentals of physics and chemistry related to this subject. In particular, the understanding of the interaction between atoms/ions has motivated the development of the concept of chemical bonding, which is the central point to describe various phenomena occurring in nature, such as the effect of doping crystals with rare earth ions on the nucleation of luminescent sites, ${ }^{3,4}$ quantum confinement in nanowires, ${ }^{5}$ the production of carbon nanotubes, ${ }^{6}$ the stoichiometry of colloidal nanocrystals ${ }^{7}$ and the effect of substituent on the structural properties of matter. ${ }^{8,9}$

The first studies ${ }^{10-12}$ on how electrons are distributed between two chemical species involved in a chemical bond gave raise the concept of covalence. Pauling was the first to define a quantitative procedure to analyze the ionicity and covalence of a chemical bond, by introducing the concept of electronegativity as "the power of an atom, in a molecule,

*e-mail: heveson.matos@ufob.edu.br to attract electrons to itself". ${ }^{1,13}$ Pauling formulated the electronegativity scale based on an empirical relationship involving the simple binding energy of the interacting species in a molecule. Together with thermochemical data, he estimated the electronegativity of various chemical elements.

Although Pauling was successful in setting up an empirical scale and interpreted with the help of quantum mechanics, the electronegativity is not a concept with an exact quantitative definition. Thus, in 1935, Mulliken ${ }^{2}$ introduced a new absolute scale of electronegativity based on electron affinity, ionization potential and molecular orbital theory. The dependence of the electronegativity on the orbital characteristics of an atom in a molecule brings a more secure theoretical foundation to the electronegativity concept, as emphasized by Coulson et al. ${ }^{14}$ There are publications which tabulate data and show different ways of discussing Mulliken's electronegativity. ${ }^{15-18}$

Despite the simplicity of the Mulliken's scale, there are some difficulties in applying it to most chemical elements quantitatively. The main difficulty is the lack of reliable information on electron affinities. Based on different physical-chemistry arguments, several scales of electronegativity were developed, such as Gordy's, ${ }^{19}$ 
Sanderson's, ${ }^{20}$ Allred-Rochow's, ${ }^{21}$ Allen's ${ }^{22}$ and RahmHoffmann ${ }^{23}$ scales. In addition, there are electronegativity equalization methods (EEM), which establish that the bonded chemical species have their electronegativities equalized. ${ }^{24-32}$

By the experimental side, methods of measuring electronegativity are based on thermochemical techniques, ${ }^{33,34}$ and atomic force microscopy (AFM). In the last years, AFM has been widely used in chemical applications, such as measurement of short-range chemical forces and discrimination of the Pauling bond order. ${ }^{35-38}$

The electronegativity difference $(\Delta \chi)$ of two atoms is a quantitative indication of the amount of electronic charge transferred when a chemical bond occurs. Consequently, the amount of charge transferred gives a plausible indication of the character of the chemical bond. In fact, $\Delta \chi$ should depends on the amount of charge transferred between the outermost orbitals of the two chemical species involved in the bond. A relationship between the charge transferred and $\Delta \chi$ was developed by Parr and Pearson. ${ }^{17,39,40}$ However, three points still persist: $(i)$ most scales do not cover all chemical elements; (ii) electronegativity difference is available only for diatomic compounds; and (iii) Par and Pearson's model has a dependence on electron affinity, an observable not easy to obtain. In particular, a simple relationship between $\Delta \chi$ and charge ge ( $\mathrm{g}$ is the charge factor, the fraction of the electronic charge involved in the chemical bond, and e the elementary charge) is of fundamental importance in the study of lanthanide-doped ionic solids. Spectroscopic properties may be obtained most easily by the relationship involving the charge transferred in the bonding and its structural properties.

Thus, by using the very basic Coulomb interaction and the direct proportionality between the electric dipole moment of the bond $(\mu)$ and $\Delta \chi$, this work aims to give an alternative analytical expression to $\Delta \chi$ of binary ionic solids. The final equation to $\Delta \chi$ is a function of the charge ge, the packing factor (p) and the effective atomic number $\left(\mathrm{Z}_{\text {eff }}\right) .{ }^{41}$ The direct relationship between $\Delta \chi$ and ge give us a new insight to understand the ionic chemical bond. This expression can be applied to calculate $\Delta \chi$ of all binary ionic solids. A comparison with the scales of Pauling, ${ }^{1,13}$ Gordy, ${ }^{19}$ Allred-Rochow ${ }^{21}$ and Allen ${ }^{22}$ is carried out.

\section{Methodology}

\section{Effective atomic number of ionic diatomic systems}

The effective atomic number $\left(Z_{\text {eff }}\right)$ of polyatomic compounds is a physical quantity which describes the average number of protons in a polyatomic compound.
Recently, Lima and Couto dos Santos $^{41}$ considered the interaction between $\mathrm{A}$ and $\mathrm{B}$ chemical species in binary solids with dominant ionic character as a dominant Coulomb type interaction, $\mathrm{U}$. There, the authors considered that the metal to non-metal interaction occurs through a mainly $\sigma$-type bond in the cation-anion direction, which means that $\mathrm{U}$ can be treated only with radial dependence. ${ }^{42}$ Further, $U$ must depend on the atomic number of the interacting species and is modulated by the packing factor, $p$, because the change of the size of the chemical species involved in the ionic bonding is due to the charge transfer. $\mathrm{p}$ is obtained through a similar expression used in solid state physics, but now using both crystalline and ionic radii of the interacting species. This is to be highlighted, because it is usual to find p only for monoatomic solids. ${ }^{43}$ Thus, $p$ is calculated based on the type of structure and the number of occupancy $\left(\mathrm{n}_{\mathrm{i}}\right)$ of cations and anions (assumed as compacted spheres), which means the volume of each ion inside the unit cell. The contribution of each ion is taken into account separately to calculate the volume fraction of each sphere. For polyatomic systems, $\mathrm{p}$ is a summation of all fractions. The effective potential, $\mathrm{U}_{\text {eff }}$, depends on $\mathrm{Z}_{\text {eff }}$. Such reasoning was applied to reproduce $\mathrm{Z}_{\text {eff }}$ of a series of ionic compounds successfully. There, ${ }^{41} \mathrm{p}$ had an initial interpretation: a fraction of matter, which absorbs the incident radiation. Here, $\mathrm{p}$ is a measure of the changing of the ionic size: the cation/anion enhances/quenches its own effective atomic number which leads to quench/enhance its own size, respectively. This is a way of introducing quantum mechanical correction to the Coulomb potential. By taking into account the considerations above and following the steps described in literature ${ }^{41}$ strictly, one has:

$\mathrm{p}=\frac{4 \pi}{3 \mathrm{~V}_{\mathrm{c}}} \sum_{\mathrm{i}} \mathrm{n}_{\mathrm{i}} \mathrm{R}_{\mathrm{i}}^{3}$

and

$\mathrm{Z}_{\text {eff }}=\left(\mathrm{p} \mathrm{Z}_{\mathrm{A}} \mathrm{Z}_{\mathrm{B}}\left(\frac{\overline{\mathbf{R}}_{\mathrm{AB}}}{\mathrm{R}_{\mathrm{AB}}}\right)\right)^{0.5}$

where $Z_{A}$ and $Z_{B}$ are atomic numbers of the interacting species, $\mathrm{R}_{\mathrm{i}}$ is the crystalline/ionic radius, $\mathrm{V}_{\mathrm{c}}$ is the volume of the unit cell and $n_{i}$ is the occupancy number of the $i^{\text {th }}$ ion in the unit cell. $\overline{\mathbf{R}}_{\mathrm{AB}}$ is the interatomic distance, ${ }^{44}$ which is the sum of the ionic (or crystalline) radii of the interacting ions, $\mathrm{R}_{\mathrm{AB}}$ is the effective distance between the negative and positive center of charge.

Formally, in an ionic bond at least one electron is transferred from the metal atom to the non-metal atom. This electron leaves empty an outer s, $d$ or f orbital in the metal atom to occupy an empty and outer $2 p$ orbital in the 
non-metal atom. The separation between the position of the positive center of charge (empty s, $d$ or f orbital) in the metal atom and the position of the negative center of charge (filled $2 p$ orbital) in the non-metal atom give us an insight over what $R_{A B}$ effective distance is. $R_{A B}$ is related to $Z_{\text {eff }}$ by equation 2. By using the LiF crystal as an example, the sole $2 \mathrm{~s}$ electron of the $\mathrm{Li}$ atom is completely transferred to the $3 \mathrm{p}^{5}$ orbital of the $\mathrm{F}$ atom. Thus, the $\mathrm{Li}^{+}$ion hosts a positive center of charge (empty $2 \mathrm{~s}$ orbital) and the $\mathrm{F}^{-}$ion hosts a negative center of charge (filled $2 p$ orbital). $R_{A B}$ is the radial separation between them, as shown in Figure $1 . R_{A B}$ as well as equation 2 have been already postulated. ${ }^{41}$ Even though it is applied here for binary ionic solids, equation 2 was developed to reproduce the effective atomic number of any ionic polyatomic compounds in the energy range of the photoelectric effect.

\section{Electronegativity difference in ionic solids}

In 1933, Malone ${ }^{45}$ studied the chemical bond between the $A$ and $B$ interacting species using the following relation:

$\mu=\left|\chi_{\mathrm{A}}-\chi_{\mathrm{B}}\right|$

$\mu$ is the electric dipole moment of the bond in Debye units and $\left|\chi_{A}-\chi_{B}\right|$ is the electronegativity difference of the interacting species in the Pauling scale. The standard unit in the International System of Units (SI) for the dipole moment is the Debye $\left(3.336 \times 10^{-30} \mathrm{C} \mathrm{m}\right)$. One Debye (1D) is the magnitude of the electric dipole moment of two charges of opposite sign, with magnitude of $10^{-10}$ e.s.u, separated by $1 \AA$.

Equation 3 has been considered very approximately in quantitative calculations of electronegativity ${ }^{46}$ However, if the molecule has no inversion center, the electric dipole moment term is dominant. In the case of a charge distribution with inversion center, the magnetic dipole and electric quadrupole moments contributions are dominant, but the electric dipole moment of each bond is still there. This can be verified by the multipole expansion of the interaction potential. ${ }^{47}$ Furthermore, lone-pair electrons may possess markedly asymmetric charge distributions, which gives a meaningful contribution to the electric dipole moment. One example is the $\mathrm{NH}_{3}$ molecule, which has $1.5 \mathrm{D}$ as the net electric dipole moment. ${ }^{33}$ In fact, the asymmetric charge distribution contributes significantly to the net electric dipole moment, although the $\mathrm{N}-\mathrm{H} \sigma$ bond is not polar enough. In this way, equation 3 can be used with good approximation to calculate $\Delta \chi$ of an ionic solid possessing or not an asymmetric charge distribution. We pictured this frame (the $\mathrm{A}-\mathrm{B}$ chemical bond) as an electric dipole moment, $\mu$, formed by two opposite charges of equal magnitude, separated by a distance $R_{A B}$. Thus, making the electric dipole moment explicit, equation 3 can be rewritten as:

$\Delta \chi=\frac{\mu}{\mathrm{D}}=\frac{\mathrm{geR}_{\mathrm{AB}}}{\mathrm{D}}$

where $\mathrm{g}$ is the charge factor, ge is the charge devoted to the A-B bond (the effective charge participating in the chemical bond), the fraction of the electronic charge devoted to the bond, e is the elementary charge, and $\mathrm{D}$ is the Debye constant. Debye's constant is explicit in equation 4, because $\Delta \chi$ is being expressed in Pauling units. Other types of conversion to Pauling's scale has been carried out elsewhere. ${ }^{23,48}$

By rewriting equation 2 in order to express the relation between $\overline{\mathbf{R}}_{\mathrm{AB}}$ and $\mathrm{R}_{\mathrm{AB}}$, one has:

$\mathrm{R}_{\mathrm{AB}}=\left(\frac{\mathrm{pZ} \mathrm{Z}_{\mathrm{A}} \mathrm{Z}_{\mathrm{B}}}{\mathrm{Z}_{\mathrm{eff}}^{2}}\right) \overline{\mathbf{R}}_{\mathrm{AB}}$

Equation 5 makes it clear that the relationship between effective distance and interatomic distance is through the

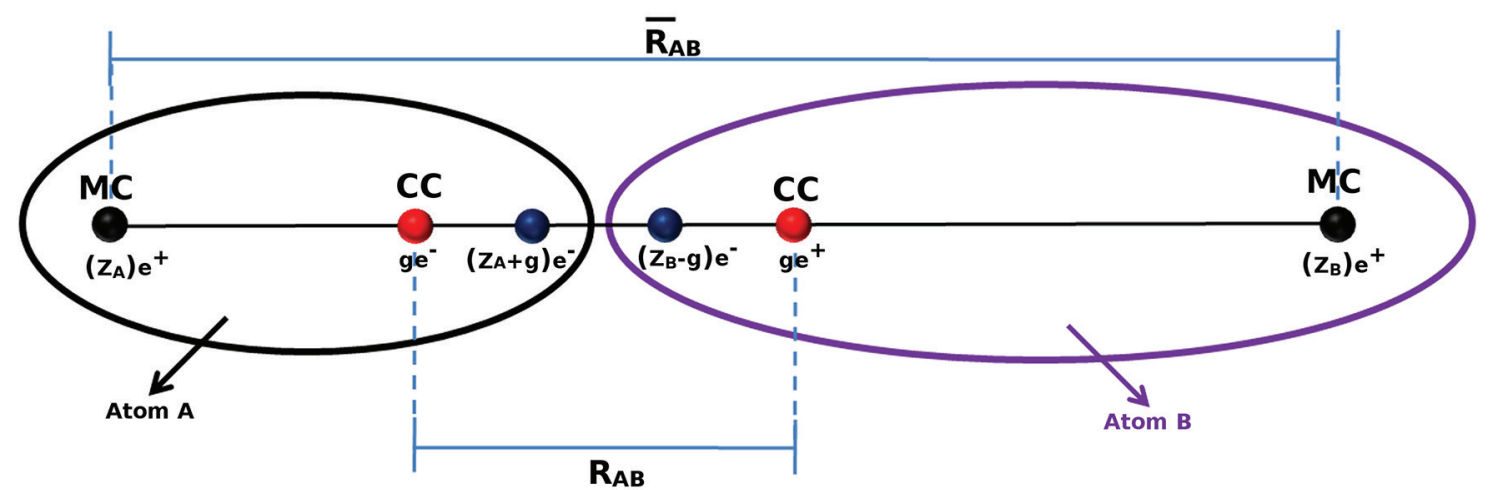

Figure 1. Mass center (MC) and charge center $(C C)$ of the $A$ and $B$ interacting species. $Z_{A} e^{+}$and $Z_{B} e^{+}$are its nuclear charges, respectively. $R_{A B}$ is the effective distance between the negative $\left(\mathrm{ge}^{-}\right)$and positive $\left(\mathrm{ge}^{+}\right)$charge centers. $\overline{\mathbf{R}}_{\mathrm{AB}}$ is the internuclear separation. $\left(\mathrm{Z}_{\mathrm{A}}+\mathrm{g}\right) \mathrm{e}^{-}$and $\left(\mathrm{Z}_{\mathrm{B}}-\mathrm{g}\right) \mathrm{e}^{-}$are the total electronic charges around the species. 
correction factor (in parentheses). This correction factor depends on intrinsic peculiarities of the ionic solid under consideration, namely, $\mathrm{p}, \mathrm{Z}_{\mathrm{A}}, \mathrm{Z}_{\mathrm{B}}, \mathrm{Z}_{\text {eff }}$. It is important to highlight that $\overline{\mathbf{R}}_{\mathrm{AB}}$ can never be equal to $\mathrm{R}_{\mathrm{AB}}$, because the center of charge can never coincide with the center of mass of the ions (see Figure 1) when a chemical bond takes place. A similar comprehension was already used some decades ago, ${ }^{49,50}$ and gave a decisive improvement around the interpretation of the crystal field effect on the luminescence behavior of lanthanide containing compounds.

By combining equations 4 and 5 , we obtain

$\Delta \chi=\left(\frac{\mathrm{p} \mathrm{Z}_{\mathrm{A}} \mathrm{Z}_{\mathrm{B}}}{\mathrm{Z}_{\mathrm{eff}}^{2}}\right) \frac{\mathrm{ge} \overline{\mathbf{R}}_{\mathrm{AB}}}{\mathrm{D}}$

As one could expect, the electronegativity difference appears directly proportional to the interaction charge devoted to the $\mathrm{A}-\mathrm{B}$ bond. The magnitude of $\Delta \chi$ is a measure of the amount of charge transferred when the chemical bond is formed. $\Delta \chi$ is directly proportional to $\mathrm{p}, \mathrm{Z}_{\mathrm{A}} \mathrm{Z}_{\mathrm{B}}$ and $\overline{\mathbf{R}}_{\mathrm{AB}}$ (A and $\mathrm{B}$ stand for the anion and cation, respectively), and inversely proportional to the $Z_{\text {eff }}^{2}$. Thus, an alternative analytical expression to obtain the electronegativity difference of any ionic compound, with no need of numerical procedures, is being presented.

\section{Results and Discussion}

In order to use equation 6 , a detailed analysis of $Z_{\text {eff }}$, $\mathrm{p}$ and $\mathrm{g}$ has to be developed. Table 1 shows the values of $\mathrm{Z}_{\text {eff }}$ and $\mathrm{p}$ for some binary compounds using the crystal radii. ${ }^{41}$ Interested readers should refer to literature ${ }^{41}$ for more detailed calculations. The crystal and ionic radii with respect to the coordination number $(\mathrm{CN})$, taken from literature ${ }^{44}$ are also shown.

\section{Charge factor}

The greatest difficulty in applying the present model more widely is to obtain $\mathrm{g}$. In fact, there are few $\mathrm{g}$ values available. In related literature $g$ values for binary systems have been calculated through different methods (ab initio, density functional theory (DFT) and the natural bond orbital (NBO) or Mulliken population charges). As exceptions to the rule, the $\mathrm{LiF}$ and $\mathrm{NaF}$ fluorides have some values of $\mathrm{g}$ available. In these cases, an average charge factor has been used. For the $\mathrm{CaF}_{2}$ and $\mathrm{SiO}_{2}$ compounds we have used $\mathrm{g}$ taken from $\mathrm{CaF}$ and $\mathrm{SiO}$ non stoichiometric molecules, respectively. The values of $g$ are shown in Table 2.

\section{Oxides}

Equation 6 has been applied to 6 oxides: $\mathrm{BeO}, \mathrm{Al}_{2} \mathrm{O}_{3}$, $\mathrm{MgO}, \mathrm{SiO}_{2}, \mathrm{ZnO}$ and $\mathrm{Eu}_{2} \mathrm{O}_{3}$. Expect for $\mathrm{ZnO}$ and $\mathrm{SiO}_{2}$, the electronegativity difference is greater than 2 as shown in Table 2 and Figure 2. Except for the $\mathrm{Eu}_{2} \mathrm{O}_{3}$ system, all values were taken from literature.

For the $\mathrm{Eu}_{2} \mathrm{O}_{3}$ system, the charge factor has been obtained by using the simple overlap model (SOM),$^{50}$ the Batista-Longo improved model (BLIM) ${ }^{57}$ and the method of equivalent nearest neighbors (MENN).$^{58}$ These three models are well known in crystal field calculations for lanthanides containing systems. By saying few words on the models to guide the reader, the first introduces the contribution of the covalence of an ionic bond and reproduces very confidently the crystal field levels of lanthanide containing materials, the second makes explicit the dependence of the Eu ion effective charge with the radial distance, and the third, based on the SOM, uses point group symmetry and electrostatic equilibrium to obtain $\mathrm{g}$. This latter also explains the high coordination number $(\mathrm{CN})$ and the weakness of the crystal

Table 1. Benchmark values to obtain the electronegativity ${ }^{41,44}$

\begin{tabular}{|c|c|c|c|c|}
\hline Structure & $Z_{\text {eff }}^{a}$ & $\mathrm{p}^{\mathrm{a}}$ & $\mathrm{CR}-\mathrm{CN}^{\mathrm{b}} / \AA$ & $\mathrm{AR}-\mathrm{CN}^{\mathrm{b}} / \AA$ \\
\hline $\mathrm{BeO}$ & 7.176 & 0.600 & 0.41-IV & $1.25-\mathrm{IV}$ \\
\hline $\mathrm{LiF}$ & $7.999(7.68)^{\mathfrak{c}}$ & $0.624(0.721)^{\mathrm{c}}$ & $0.9(0.76)-\mathrm{VI}$ & 1.19(1.33)-VI \\
\hline $\mathrm{Al}_{2} \mathrm{O}_{3}$ & 13.786 & 0.595 & $0.675-\mathrm{VI}$ & $1.22-\mathrm{III}$ \\
\hline $\mathrm{MgO}$ & 13.683 & 0.580 & $0.86-\mathrm{VI}$ & $1.26-\mathrm{VI}$ \\
\hline $\mathrm{NaF}$ & $14.372(13.251)^{\mathrm{c}}$ & $0.524(0.551)^{\mathrm{c}}$ & $1.16(1.02)-\mathrm{VI}$ & 1.19(1.33)-VI \\
\hline $\mathrm{SiO}_{2}$ & 9.958 & 0.344 & 0.4-IV & $1.21-\mathrm{II}$ \\
\hline $\mathrm{CaF}_{2}$ & $19.952(19.086)^{\mathrm{c}}$ & $0.532(0.604)^{\mathrm{c}}$ & $1.26(1.12)-\mathrm{VIII}$ & 1.17(1.31)-IV \\
\hline $\mathrm{ZnO}$ & 24.636 & 0.573 & 0.88 -VI & $1.26-\mathrm{VI}$ \\
\hline
\end{tabular}

${ }^{a}$ Theoretical $Z_{\text {eff }}$ and $p$ obtained from literature; ${ }^{41}$ bthe crystal radii as well as the cation (CR) and anion (AR) radii with respect to the coordination number $(\mathrm{CN})$ were taken from literature; ${ }^{44}$ cor the $\mathrm{LiF}, \mathrm{NaF}$ and $\mathrm{CaF}_{2}$ compounds, it is also shown data obtained with the ionic radii (in parentheses). $\mathrm{Z}_{\text {eff }}$ : the effective atomic number; p: packing factor. 
Table 2. Charge factors and electronegativity differences

\begin{tabular}{|c|c|c|c|c|c|c|}
\hline Structure & $\mathrm{g}$ & $\Delta \chi_{\mathrm{H}} /$ Pauling unit & $\Delta \chi_{\mathrm{P}} /$ Pauling unit & $\Delta \chi_{\mathrm{G}} /$ Pauling unit & $\Delta \chi_{\mathrm{AR}} /$ Pauling unit & $\Delta \chi_{\mathrm{A}} /$ Pauling unit \\
\hline $\mathrm{BeO}$ & $0.62^{\mathrm{a}}$ & 2.18 & 2 & 2 & 2.03 & 2.034 \\
\hline $\mathrm{LiF}$ & $0.90^{\mathrm{a}, \mathrm{b}, \mathrm{c}}$ & $2.40(3.01)$ & 3 & 3 & 3.13 & 3.281 \\
\hline $\mathrm{Al}_{2} \mathrm{O}_{3}$ & $0.63^{\mathrm{d}}$ & 2.18 & 2 & 1.95 & 2.14 & 1.997 \\
\hline $\mathrm{MgO}$ & $0.72^{\mathrm{a}}$ & 2.20 & 2.3 & 2.25 & 2.27 & 2.317 \\
\hline $\mathrm{NaF}$ & $0.92^{\mathrm{a}, \mathrm{b}}$ & $2.63(3.26)$ & 3.1 & 3.05 & 3.18 & 3.324 \\
\hline $\mathrm{SiO}_{2}$ & $0.55^{\mathrm{e}}$ & 1.67 & 1.7 & 1.65 & 1.76 & 1.694 \\
\hline $\mathrm{CaF}_{2}$ & $0.78^{\mathrm{b}}$ & $2.21(2.74)$ & 3 & 2.95 & 3.06 & 3.159 \\
\hline $\mathrm{ZnO}$ & $0.80^{\mathrm{f}}$ & 1.88 & 1.9 & 2.25 & 1.84 & 2.01 \\
\hline $\mathrm{Eu}_{2} \mathrm{O}_{3}$ & 0.60 & 2.10 & 2.3 & - & - & - \\
\hline
\end{tabular}

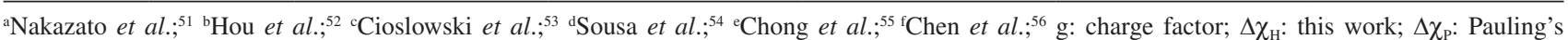
electronegativity; $\Delta \chi_{\mathrm{G}}$ : Gordy's electronegativity; $\Delta \chi_{\mathrm{AR}}$ : Allred-Rochow difference; $\Delta \chi_{\mathrm{A}}$ : Allen's electronegativity. For the $\mathrm{LiF}, \mathrm{NaF}$ and $\mathrm{CaF}{ }_{2}$, the values calculated with the ionic radius are shown in parentheses.

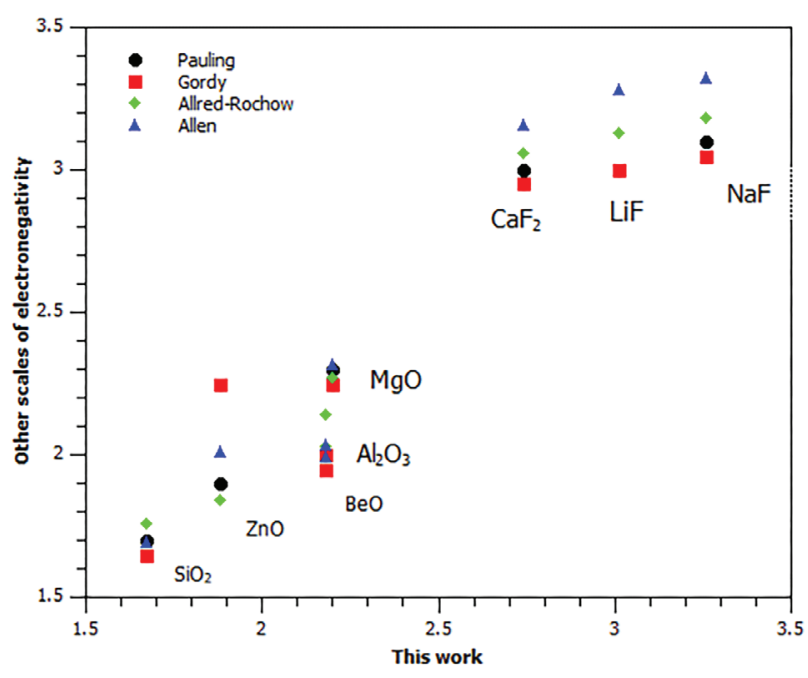

Figure 2. Comparison between the electronegativity difference of this work with that of Pauling, ${ }^{1,13}$ Gordy, ${ }^{19}$ Allred-Rochow ${ }^{21}$ and Allen ${ }^{22}$ for binary ionic solids.

field perturbation of the lanthanide ion site..$^{59}$ The authors encourage interested readers to consult the literature. ${ }^{59}$

The BLIM has been developed for a series of europium coordination compounds with eight nearest neighbours $(\mathrm{NN})$. However, the shielding of the $4 \mathrm{f}$ orbitals by the $5 \mathrm{~s}$ and $5 \mathrm{p}$ filled shells is valid no matter the host, which leads to very similar energy level scheme of the europium ion no matter the host, and the splitting of levels only depends on the local symmetry. Thus, in any inorganic compound the europium effective charge must be similar, as one can see in literature,${ }^{60}$ in which the Eu ions are at least in

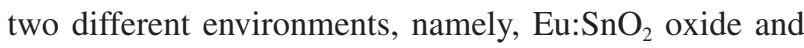
$\mathrm{Eu}: \mathrm{BaLiF}_{3}$ fluoride. In the $\mathrm{Eu}_{2} \mathrm{O}_{3}$ crystal, as in the $\mathrm{SnO}_{2}$, the $\mathrm{CN}$ is six. Using the MENN, in order to estimate a reasonable range for $\mathrm{g}$, the minimum value is obtained by dividing the $\mathrm{Eu}$ ion valence by 6 , to give $\mathrm{g}_{\min }=0.5$. The maximum is obtained by calculating the Eu ion effective charge through the BLIM model at the $\mathrm{Eu}-\mathrm{O}$ middle distance and dividing it by 6 to give $g_{\max }=0.708$. The charge factor range is very similar to that obtained for oxide and fluoride crystals $(0.5 \leq \mathrm{g} \leq 0.8)^{58,60,61}$ as well as comparable to the effective charges obtained by the radial effective charge model. ${ }^{62,63}$ By using an average charge factor of 0.604 , we obtain $\Delta \chi=2.1$, with a relative error around $9 \%$ in comparison to the Pauling's electronegativity difference. Thus, we postulate that $\mathrm{g}$ can be interpreted as the amount of charge transferred to around the middle metal-ligating ion distance, closer to the ligating ion $\left(\mathrm{g}<\mathrm{g}_{\max }\right)$, when the chemical bond takes place.

\section{Fluoride compounds}

In fluoride systems, equation 6 has been tested for three materials: $\mathrm{LiF}, \mathrm{NaF}$ and $\mathrm{CaF}_{2}$. As the fluorine is the most electronegative element, the electronegativity difference between $\mathrm{M}-\mathrm{F}$ ( $\mathrm{M}$ stands to the metal atom) is significantly greater than oxides systems (see Table 2 and Figure 2). We have carried out calculations using both ionic and crystal radii and the charge factor follows the same growth trend.

Sensitivity of the electronegativity to the ionic radius in fluoride systems

Specifically for the fluoride systems, we performed calculations using both crystal and ionic radii. However, the use of the crystal radius in the $Z_{\text {eff }}$ and $p$ calculations leads to similar estimates to that obtained for the oxides. By using the ionic radius, our estimates deviate satisfactorily in the sense of obtaining $\Delta \chi_{\mathrm{P}}$, which are less than 1, 6 and $9 \%$ for $\mathrm{LiF}, \mathrm{NaF}$ and $\mathrm{CaF}_{2}$, respectively, as shown in Figure 2. 
In comparison to Allen's scale, relative errors are less than 9,2 and $14 \%$, respectively.

By using the crystalline radius, we obtained a relative error in a range of 15 and $30 \%$ in comparison to the listed scales, for the same series. A similar behavior has also been obtained in the $\mathrm{Z}_{\text {eff }}$ calculations for these fluorides. However, the relative error was less than $20 \%$ in comparison to $Z_{\text {eff }}$ experimental data obtained in the photoelectric effect region. In addition, the deviation between the $Z_{\text {eff }}$ obtained with the crystalline and ionic radii is less than $5 \%$. Even though the crystalline radius corresponds more closely to the physical size of the ions in a solid, since it varies very little from structure to structure. ${ }^{44}$

The ionic radius has led to values closer to those in related literature for bonds with higher ionicity, as it is the case of the fluorides. This behavior can be explained by the fact that the anion radius in fluorides is higher than the covalent radius. The increasing repulsion between the electrons during the process of formation of the compound causes their spreading in space. Under such circumstances, the electron do not stay in the original atom, because the oxidation of the metal atom and the reduction of the non metal atom is the way of stabilizing the chemical species by getting the electronic configuration of a noble gas. Therefore, there is a greater amount of charge transferred in fluorides than in oxide systems, as it can be noted through the magnitude of $\mathrm{g}$. That is, more space is needed to the electronic cloud around the $\mathrm{F}^{-}$nucleus. Therefore, our estimates are closer to all scales by using the ionic radius in the calculations of $Z_{\text {eff }}$ and $p$. The use of ionic radius is, then, physically acceptable and seems to describe the properties of these fluoride compounds more adequately, due to a high degree of ionicity. Thus, the distance between the negative and positive center of charge plays an important rule to justify the spread of each electron transferred in chemical bonding.

\section{Comparison with other scales}

Figure 2 shows a comparison between all electronegativity differences treated here. In the horizontal axis one can see $\Delta \chi$ obtained with equation 6 . This scale presents the same trends as in most other scales in the literature. Allen's scale has been converted to the Pauling scale adequately. ${ }^{23,45}$

In Figure 2 one can also note that the relative errors of $\Delta \chi$ are around $10 \%$ in comparison with those of Pauling, ${ }^{1,13}$ Gordy, ${ }^{19}$ Allred-Rochow ${ }^{21}$ and Allen. ${ }^{22}$ For the $\mathrm{CaF}_{2}$ fluoride, our achievements show an average relative error of $10 \%$. However, the $\mathrm{CaF}$ non stoichiometric system has not the same charge distribution of the $\mathrm{CaF}_{2}$ crystal. This explains such mismatch. In contrast, we can do one more analysis about the charge transferred in the $\mathrm{Ca}-\mathrm{F}$ bonding using the octet rule. Note that the two 4 s electrons (calcium atom) is completely transferred to the $2 \mathrm{p}$ orbital (of both fluorine atoms). Therefore, the charge factor of the $\mathrm{Ca}-\mathrm{F}$ bond should be 1 . Using this argument and $\mathrm{Z}_{\mathrm{eff}}$ and $\mathrm{p}$ calculated with the ionic radius, we obtained $\Delta \chi=2.84$, meaning a relative error of $5.5 \%$ compared to Pauling's scale.

Electronegativity is a concept related to reactivity and charge transfer between atoms and specify ionic or covalent character of the final compound. In our calculations, which can be used to any ionic solid, we have noted that, in fluoride compounds, entering the ionic radii, not the crystalline radii, $\Delta \chi$ is best reproduced. At this moment, our interpretation is: the greater the charge factor, the great the space around the ion to house it. We end by highlighting that understanding electronegativity is still a challenge, including obtaining the $\mathrm{g}$ factor. Equation 6 is a contribution on how ions organize themselves in a solid, through the packing factor $\mathrm{p}$, and how electrons are transferred in the process of ionic chemical bonding, through $\mathrm{g}$.

\section{Conclusions}

In summary, we are announcing an alternative analytical expression to calculate the electronegativity difference $(\Delta \chi)$ of any binary ionic solid, by postulating a relationship between $\Delta \chi$, the effective charge (ge) involved in the bond and the effective atomic number $\left(\mathrm{Z}_{\text {eff }}\right)$. This expression was applied to nine binary compounds, namely, $\mathrm{BeO}, \mathrm{LiF}$, $\mathrm{Al}_{2} \mathrm{O}_{3}, \mathrm{MgO}, \mathrm{NaF}, \mathrm{SiO}_{2}, \mathrm{CaF}_{2}, \mathrm{ZnO}$ and $\mathrm{Eu}_{2} \mathrm{O}_{3}$. Deviations around $10 \%$ were obtained by using the charge factor, $\mathrm{g}$, taken from $a b$ initio or phenomenological procedures in comparison to four electronegativity scales, namely, Pauling, Gordy, Allred-Rochow and Allen. We have also shown that $\Delta \chi$ increases with $g$, as expected. $g$ is postulated as the amount of charge transferred from the metal to the non-metal atoms when the chemical bond occurs. A correcting factor to $\overline{\mathbf{R}}_{\mathrm{AB}}$ and $\Delta \chi$, which depends on $\mathrm{p}, \mathrm{Z}_{\mathrm{A}}, \mathrm{Z}_{\mathrm{B}}$, $\mathrm{Z}_{\text {eff }}$ has been introduced. The values of $\mathrm{g}$, the fraction of the electron transferred, play the important role of indicating the ionic character of the chemical bond. Our model shows that a large $g$ means a large $\Delta \chi$ and, consequently, greater ionic character. The satisfactory calculations mean a strong indication that our expression can be used to determine $\Delta \chi$ in any binary ionic compounds (not only for diatomic molecules and including all chemical elements). The challenge remaining is to find an analytical way of obtaining the charge factor. 


\section{Acknowledgments}

The authors strongly acknowledge the financial support from Inct-INAMI, CNPq, CAPES and FAPITEC Brazilian agencies. Heveson Lima is grateful to Dr Afranio Sousa for valuable discussions and support on data presentation.

\section{References}

1. Pauling, L.; The Nature of Chemical Bond and the Structure of Molecules and Crystals: An Introduction to Modern and Structural Chemistry, $3^{\text {rd }}$ ed.; Cornell University: New York, USA, 1960.

2. Mulliken, R. S.; J. Chem. Phys. 1935, 3, 573.

3. Chen, X. Y.; Sun, C. T.; Wu, S. X.; Xue, D. F.; Phys. Chem. Chem. Phys. 2017, 19, 8835.

4. Lima, H.; de Oliveira, H.; Bidault, C. C. X.; dos Santos, T. S.; Chaussedent, S.; Couto dos Santos, M. A.; J. Non-Cryst. Solids 2016, 448, 62 .

5. Sanchez-Soares, A.; Greer, J. C.; Nano Lett. 2016, 16, 7639.

6. Peng, B.; Govind, N.; Apra, V.; Klemm, M.; Hammond, J. R.; Kowalski, K.; J. Phys. Chem. A 2017, 121, 1328.

7. Sluydts, M.; de Nolf, K.; van Speybroeck, V.; Cottenier, S.; Hens, Z.; ACS Nano 2016, 10, 1462

8. James, A. M.; Laconsay, C. J.; Galbraith, J. M.; J. Phys. Chem. A 2017, 121, 5190.

9. Szatylowicz, H.; Jezuita, A.; Ejsmont, K.; Krygowski, T. M.; J. Phys. Chem. A 2017, 121, 5196.

10. Lewis, G. N.; J. Am. Chem. Soc. 1916, 38, 762.

11. Langmuir, I.; J. Am. Chem. Soc. 1919, 41, 868.

12. Heitler, W.; London, F.; Z. Phys. 1927, 4, 455.

13. Pauling, L.; J. Am. Chem. Soc. 1932, 54, 3570.

14. Coulson, C. A.; Redei, L. B.; Stocker, D.; Proc. R. Soc. London, Ser. A 1962, 270, 357.

15. Cardenas, C.; Heidar-Zadeh, F.; Ayers, P. W.; Phys. Chem. Chem. Phys. 2016, 18, 25721.

16. Iczkowski, R. P.; Margrave, J. L.; J. Am. Chem. Soc. 1961, 83, 3547.

17. Parr, R. G.; Bartolotti, L. J.; J. Am. Chem. Soc. 1982, 104, 3801.

18. Ayers, P. W.; Parr, R. G.; Pearson, R. G.; J. Chem. Phys. 2006, 124, 194107.

19. Gordy, W.; Phys. Rev. 1946, 69, 604.

20. Sanderson, R. T.; Science 1955, 121, 207.

21. Allred, A. L.; Rochow, E. G.; J. Inorg. Nucl. Chem. 1958, 5, 264.

22. Allen, L. C.; J. Am. Chem. Soc. 1989, 111, 9003.

23. Rahm, M.; Zeng, T.; Hoffmann, R.; J. Am. Chem. Soc. 2019 , $141,342$.

24. Mortier, W. J.; van Genechten, K.; Gasteiger, J.; J. Am. Chem. Soc. 1985, 107, 829.
25. Mortier, W. J.; Ghosh, S. K.; Shankar, S.; J. Am. Chem. Soc. 1986, 108, 4315.

26. van Genechten, K.; Mortier, W.; Geerlings, P.; J. Chem. Soc., Chem. Commun. 1986, 1278.

27. Bultinck, P.; Langenaeker, W.; Lahorte, P.; de Proft, F.; Geerlings, P.; Waroquier, M.; Tollenaere, J. P.; J. Phys. Chem. A 2002, 106, 7887.

28. Bultinck, P.; Langenaeker, W.; Lahorte, P.; de Proft, F.; Geerlings, P.; van Alsenoy, C.; Tollenaere, J. P.; J. Phys. Chem. A 2002, 106, 7895.

29. Bultinck, P.; Vanholme, R.; Popelier, P. L. A.; de Proft, F.; Geerlings, P.; J. Phys. Chem. A 2004, 108, 10359.

30. Giese, T. J.; York, D. M.; Theor. Chem. Acc. 2012, 131, 1145.

31. Verstraelen, T.; Ayers, P. W.; van Speybroeck, V.; Waroquier, M.; J. Chem. Phys. 2013, 138, 074108.

32. Wu, Y.; Li, Y.; Hu, N.; Hong, M.; Phys. Chem. Chem. Phys. 2014, 16, 2674.

33. Pritchard, H. O.; Skinner, H. A.; Chem. Rev. 1955, 55, 745.

34. Wagman, D. D.; Evans, W. H.; Parker, V. B.; Schumm, R. H.; Halow, I.; Balley, S. M.; Churney, K. L.; Nuttal, K. L.; The NBS Tables of Chemical Thermodynamic Properties: Selected Values for Inorganic and C1 and C2 Organic Substances in SI Units, vol. 11; American Chemical Society and American Institute of Physics: Gaithersburg, USA, 1982.

35. Onoda, J.; Ondracek, M.; Jelınek, P.; Sugimoto, Y.; Nat. Commun. 2017, 8, 15155.

36. Lantz, M. A.; Hug, H. J.; Hoffmann, R.; van Schendel, P. J. A.; Kappenberger, P.; Martin, S.; Baratoff, A.; Güntherodt, H.-J.; Science 2001, 291, 2580.

37. Gross, L.; Mohn, F.; Moll, N.; Schuler, B.; Criado, A.; Guitián, E.; Peña, D.; Gourdon, A.; Meyer, G.; Science 2012, 337, 1326.

38. Pavlicek, N.; Schuler, B.; Collazos, S.; Moll, N.; Pérez, D.; Guitián, E.; Meyer, G.; Peña, D.; Gross, L.; Nat. Chem. 2015, 7, 623.

39. Parr, R. G.; Pearson, R. G.; J. Am. Chem. Soc. 1983, 105, 7512.

40. Ayers, P. W.; Faraday Discuss. 2007, 135, 161.

41. Lima, H.; Couto dos Santos, M. A.; J. Phys. Chem. Solids 2016, 96-97, 38.

42. Jørgensen, C. K.; Modern Aspects of Ligand Field Theory; North-Holland: Amsterdam, Netherlands, 1971.

43. Ashcroft, N. W.; Mermin, N. D.; Solid State Physics; Saunders College: Rochester, Great Britain, 1976.

44. Shannon, R. D.; Acta Cryst. 1976, 32, 751.

45. Malone, J. G.; J. Chem. Phys. 1933, 1, 197.

46. Atkins, P. W.; Physical Chemistry; Oxford University Press: Oxford, Great Britain, 1994.

47. Sakurai, J. J.; Modern Quantum Mechanics, $1^{\text {st }}$ ed.; AddisonWesley: New York, USA, 1994.

48. Mann, J. B.; Meek, T. L.; Allen, L. C.; J. Am. Chem. Soc. 2000, $122,2780$.

49. Malta, O. L.; Chem. Phys. Lett. 1982, 87, 27. 
50. Malta, O. L.; Chem. Phys. Lett. 1982, 88, 353.

51. Nakazato, D. T. I.; de Sa, E. L.; Haiduke, R. L. A.; Int. J. Quantum Chem. 2010, 110, 1729.

52. Hou, S.; Bernath, P. F.; J. Phys. Chem. A 2015, 119, 1435.

53. Cioslowski, J.; J. Am. Chem. Soc. 1989, 111, 8333.

54. Sousa, C.; Pacchioni, F. I.; J. Chem. Phys. 1993, 99, 6818.

55. Chong, D. P.; Chem. Phys. Lett. 1994, 220, 102.

56. Chen, M.; Straatsma, T. P.; Fang, Z.; Dixon, D. A.; J. Phys. Chem. C 2016, 120, 20400.

57. Batista, H. J.; Longo, R. L.; Int. J. Quantum Chem. 2002, 90, 924.
58. Oliveira, Y. A. R.; Lima, H.; Souza, A. S.; Couto dos Santos, M. A.; Opt. Mater. 2014, 36, 655.

59. Couto dos Santos, M. A.; Chem. Phys. Lett. 2008, 455, 339.

60. Lima, H.; Oliveira, Y. A. R.; Souza, A. S.; Gonçalves, R. R.; Couto dos Santos, M. A.; J. Lumin. 2016, 170, 556.

61. Couto dos Santos, M. A.; Europhys. Lett. 2009, 87, 67006.

62. Baldov1, J. J.; Borras-Almenar, J. J.; Clemente-Juan, J. M.; Coronado, E.; Gaita-Arino, A.; Dalton Trans. 2012, 41, 13705.

63. Baldov1, J. J.; Gaita-Arino, A.; Coronado, E.; Dalton Trans. 2015, 44, 12535. 